\title{
Detection of Vulnerable Road Users in Blind Spots through Bluetooth Low Energy
}

\author{
Jo Verhaevert \\ IDLab, Department of Information Technology \\ Ghent University-imec, Technologiepark-Zwijnaarde 15, Ghent B-9052, Belgium
}

\begin{abstract}
Around a vehicle, a blind spot is an area, which cannot be seen by the driver. Blind spot accidents are a frequently occurring problem. Annually, dozens of deaths are caused by this phenomenon despite legally compulsory prevention tools and large investments in raising awareness and developing prevention systems. Because this is not enough to reduce the number of accidents, proactive solutions must be found. This article describes the evaluation of a new blind spot system based on BLE (Bluetooth Low Energy).

In this paper, a complete system is designed with on-the-shelf products and finally realized in a proof-of-concept. The final design requirements are fixed, by figuring out where the blind spots are and which various advantages and disadvantages the existing systems have. In a further stage, the system is tested in several possible critical situations, proving that it is very robust. Vulnerable road users are indeed detected by visualization on the LCD display.
\end{abstract}

\section{INTRODUCTION}

The blind spot is a known phenomenon and annually leads to dozens of deaths. A blind spot is an area around a vehicle, that the driver cannot see. Because awareness is not enough to reduce the number of accidents, proactive solutions must be found. This can be done by investigating the different causes that lead to such accidents. By analyzing the main causes of car and truck accidents, three areas of blind spots are defined: (1) to the right of the driver's compartment, because vulnerable road users can stand behind the door, (2) diagonally to the left back and to the right back of the truck and (3) at the back of the truck.

For various reasons one can easily foresee a conclusive solution for some causes, while one will never find a solution to other causes. For example, it is impossible to design a system that will detect alcohol abuse, bad road infrastructure and difficult weather conditions. On the other hand, it is very easy to develop systems that help the driver when turning. For instance, a camera system can help the driver to have a view of places that are impossible for him to see directly. Also, one can easily warn the vulnerable road users in a blind spot by ringing an alarm when taking a turn to the right. Of course, sensitizing people about the blind spot is the first priority.

The rest of the paper is structured as follows. In Section 2, this paper describes the existing passive and active systems. In Section 3 the design of the system is discussed, whereas Section 4 describes the used hardware. The measurements with the results are handled in Section 5 and Section 6 gives the conclusions.

\section{EXISTING SYSTEMS: PASSIVE AND ACTIVE}

There are several systems on the market in order to make detection of blind spots possible. These are divided into two major groups: the passive and the active ones. A passive system does not warn the driver of a potential blind spot accident. These systems are the oldest on the market and some of them are required by law. There exists three different systems: mirrors, camera systems and a specific truck cabin design. Mirrors are mandatory and are mounted on different places of the truck, reducing the spaces with limited view. As is shown in Figure 1, in general 4 different mirrors are in use: the main mirror, the width mirror, the curb mirror and the windshield mirror.

Another passive blind spot detection system required by law in many countries is a camera system. The great advantage of these cameras is the perfect image displayed of a certain blind spot area. Alternatively, the camera takes less space and adds no additional blind spots, in contrast with mirrors. However, the complex installation can be a disadvantage. In some cases there is a computer program that analyses these images and tries to detect a road user in the blind spot [1]. A last passive system is the driver's compartment design. Here the compartment is designed in a way that the driver gets a better view of the road. Additionally, there are precautions taken in 


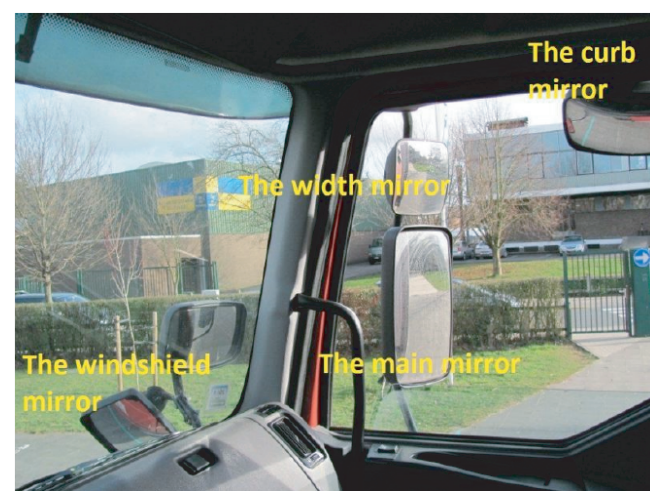

Figure 1: Different mirrors in use in a truck cabin.

case of an accident, so the vulnerable road users will not be hit or injured badly. In every passive system, it remains the responsibility of the driver and/or vulnerable road user to correctly interpret the signals.

In contrast, the active systems will warn the driver and/or vulnerable road users by means of a sound or a light signal. Lexguard, for instance, is an ultrasonic detection system, where bars mounted on the side of the truck scan for objects in the immediate vicinity [2]. Reporting is done through a sound and a light display. This system has three detection levels, with their own combination of light and sound signal. In contrast, Lisa-2-alert does not warn the driver, but the vulnerable road users [3]. As soon as the driver has a speed that is less than $30 \mathrm{~km} / \mathrm{h}$ and the direction indicator is used, there will be a light and an acoustic signal observable. In many cases those signals are forbidden during the night.

\section{DESIGN OF THE SYSTEM}

Studies have shown that it is better to warn the driver for a possible accident rather than warning the vulnerable road users, such as pedestrians and two-wheeler users. This is because a driver can react more quickly to a warning than the vulnerable road users [4].

The system is designed by using BLE (Bluetooth Low Energy), because of the overall use in recent smart phones $[5,6]$. When the proof-of-concept will be realized in an available product for the market, a lot of vulnerable users already have a smart phone with BLE enabled and only a particular application with additional software needs to be installed. When programmed as a device that continuously advertises its presence as advertisement packets on the different available channels, the receiver node interprets these advertising data such as the RSSI (Received Signal Strength Indicator) as important values.

The proof-of-concept consists of four main stages: (1) the presence of vulnerable road users is announced to the environment by advertising packets, (2) the detection nodes mounted on the truck, continuously scan for advertisement packets from the vulnerable road users, (3) after averaging over 9 different values, the RSSI values of the different road users are forwarded from the detection node to the central node and (4) in this central node in the driver's compartment, the alert levels through thresholds (no alert, mild alert and high alert) are determined for each detection node separately and visualized in a schematic map on the on-board LCD-screen, indicating the position of vulnerable road users. This is also represented on Figure 2.

\section{USED HARDWARE}

For the development of this system, the BLE modules [7] of Bluegiga, nowadays part of Silicon Laboratories, were used. There were 6 BLED112 dongles (shown in Figure 3), and one development board (as can be seen in Figure 4) provided to realize the above described proof-of-concept. All modules are fitted with the BLE112-module which consists of the BLE-stack and an 8051 microprocessor. All modules are programmed through BGScript and can be controlled through the included software (BLEGUI) dongles. The development board is used as a central node, the LCD display can easily visualize where vulnerable road users are detected. Of the six dongles, five are programmed as a detection node and one is set as the advertiser through the BLEGUI. 


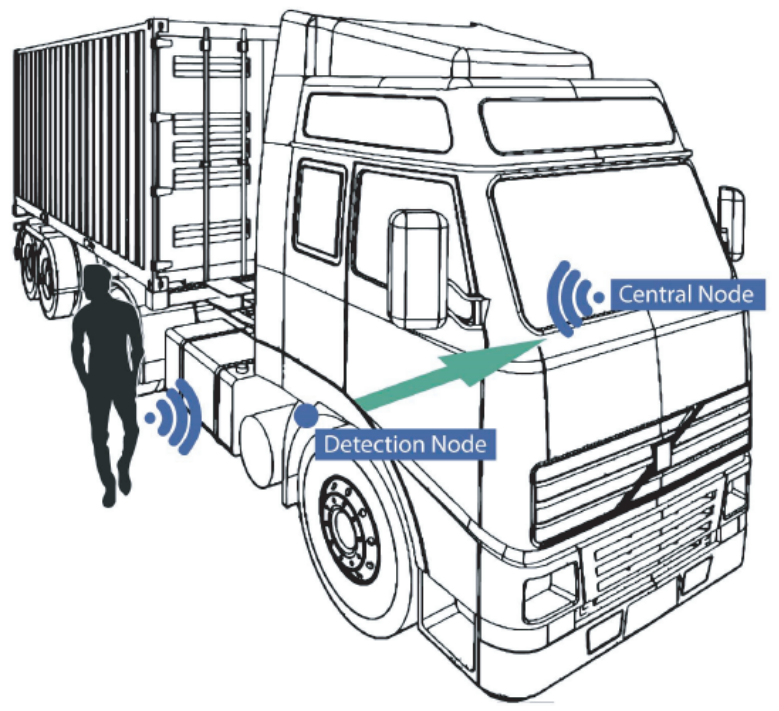

Figure 2: Graphical representation of the designed system.



Figure 3: Example of a dongle [7].

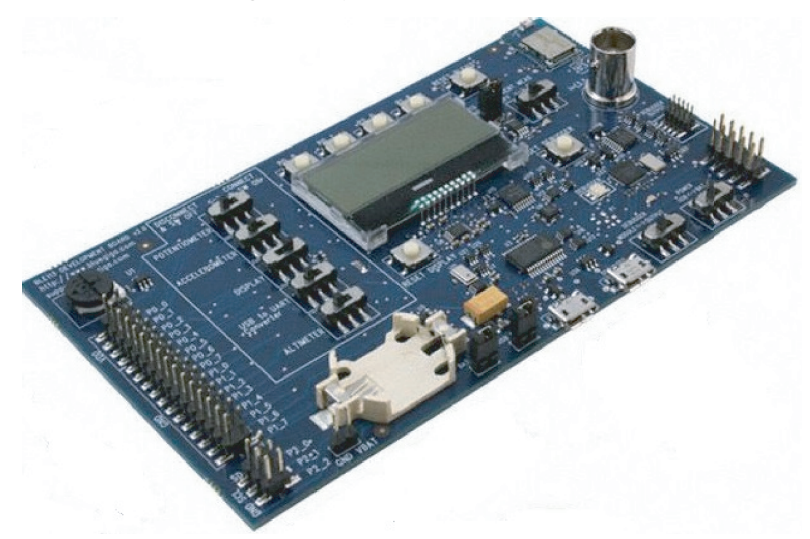

Figure 4: Example of a development board [7].

\section{MEASUREMENTS}

The system is tested in several possible critical situations, challenging the complete system. Although calculating correct distances out of these RSSI values can be very challenging [8,9], values for the different thresholds are defined based on measurements and linking ranges of RSSI values to a given distance. This results in thresholds levels 'no alert', 'mild alert' and 'high alert', which are found by experimenting. The central node can hence choose the right alert level.

For the proof-of-concept measurements, the five detection nodes were placed at an equal distance around a truck, the central node was in the driver's compartment and the vulnerable road user was also equipped with a dongle. Figure 5 gives a graphical representation of the configuration on the truck.

In order to create a system that is very stable, robust and giving a perfect detection in any possible circumstance, this system was therefore tested on different levels. In a first global measurement set-up, the system was tested in a way it should be used: five detection nodes were mounted at an equal distance around the truck and the vulnerable road users moved freely. On the LCD screen of the development board, shown in Figure 6, the 5 RSSI values of each detection node are visualized on a first line. This line is meant for debugging purposes. On a second line, the closeness of a vulnerable road user is indicated by the whole of dots and colons. The third detection node (with RSSI value of $-68 \mathrm{dBm}$ above it) indicates a road user in the far neighborhood, indicated with the collection of 3 dots as the threshold level 'mild alert'. The same holds for the fifth detection node (with RSSI value of $-59 \mathrm{dBm}$ above it). In contrast, it can be concluded that the fourth detection node with $-34 \mathrm{dBm}$ as RSSI value (with the threshold level 'high alert') shows a vulnerable road 
user in the very close vicinity. It can be concluded that vulnerable road users in the blind spot can be detected by the use of BLE.

In a second test, the reduction of the number of detection nodes was tested. There is always a possibility that a detection node is broken or that the connection with the central node fails. The system needs to keep working in that situation. Therefore the system was tested with less nodes: firstly with 1 node less (and hence 4 different detection nodes) and secondly with 2 nodes less (resulting in 3 different detection nodes). Because in both situations the detection of the vulnerable road users was perfect, this test can be concluded as perfect.

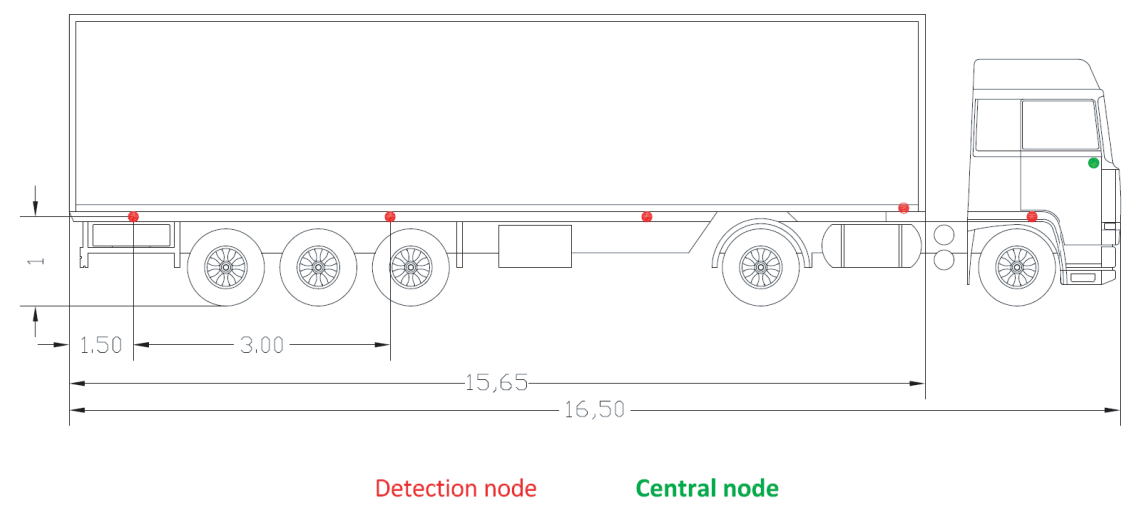

Figure 5: System set-up on a truck with trailer (in meters).

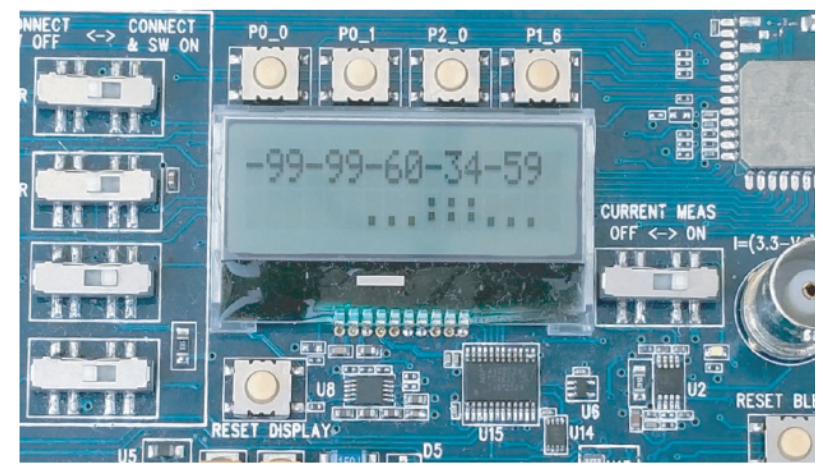

Figure 6: Measurement result with 5 detection nodes.

For the third test, the distance between the different detection nodes was increased. Augmenting the distance between neighboring detection nodes from $3 \mathrm{~m}$ to $4 \mathrm{~m}, 5 \mathrm{~m}$ and even $6 \mathrm{~m}$, resulted in similar results as described above. At the same time, it was checked that the connection between the central node in front of the truck and the fifth detection node at the back of it, was maintained.

All tests went flawless. It performed perfectly in every tested situation. It can be concluded that the proof-of-concept has reached its goal. The one disadvantage this system has, is the speed of the display. The test showed it is possible for the detection nodes to work with advertisement packets transmitted with an interval of $20 \mathrm{~ms}$, resulting in a real time detection. When the refresh rate of the display was increased, the central node started to fail.

\section{CONCLUSIONS}

This paper explains the design of a new blind spot detection system. First, different blind spots systems are described, each with its own advantages and disadvantages. In a second part the design of the system is commented, indicating that this system can have a greater value on the market. In a last part, it is described how the proof-of-concept is realized using Bluetooth Low Energy (BLE). The measurement results show that the system is working perfectly and fully complies with the imposed requirements. There is detection of vulnerable road users and the visualization on the LCD display provides plenty of information. Furthermore, the resulting system does not have the 
same problems that today's systems have. It is clear that the above described system is robust enough to work in almost every critical situation.

\section{ACKNOWLEDGMENT}

The author wants to express his gratitude to Electronics Engineering student Nick De Raeve for the fruitful cooperation and the interesting work. Without his efforts, the practical implementation described in this paper would not have been realized.

\section{REFERENCES}

1. Chen, C. T. and Y. S. Chen, "Real-time approaching vehicle detection in blind-spot area," 12th International IEEE Conference on Intelligent Transportation Systems, 24-29, St. Louis, MO, USA, Oct. 2009.

2. http://www.lexguard.nl/.

3. http://www.lisa2alert.com/.

4. Lin, J. R., T. Talty, and O. K. Tonguz, "A blind zone alert system based on intra-vehicular wireless sensor networks," IEEE Transactions on Industrial Informatics, Vol. 11, No. 2, 476$484,2015$.

5. Zhang, M., W. Xia, and L. Shen, "Bluetooth low energy based motion sensing system," Sixth International Conference on Wireless Communications and Signal Processing (WCSP2014), 1-5, Hefei, China, Oct. 2014.

6. Lin, J. R., T. Talty, and O. K. Tonguz, "On the potential of bluetooth low energy technology for vehicular applications," IEEE Communications Magazine, Vol. 53, No. 1, 267-275, 2015.

7. http://www.silabs.com/products/wireless/bluetooth/bluetooth-low-energy-modules/ble112bluetooth-smart-module.

8. Abusubaih, M., B. Rathke, and A. Wolisz, "A dual distance measurement scheme for indoor IEEE 802.11 Wireless Local Area Networks," 9th IFIP International Conference on Mobile Wireless Communications Networks, 121-125, Cork, Ireland, Sep. 2007.

9. Xu, J., W. Liu, F. Lang, Y. Zhang, and C. Wang, "Distance measurement model based on RSSI in WSN," Wireless Sensor Network, Vol. 02, No. 08, 606-611, 2010. 